\title{
ALEXANDER'S AND MARKOV'S THEOREMS IN DIMENSION FOUR
}

\author{
SEIICHI KAMADA
}

\begin{abstract}
Alexander's and Markov's theorems state that any link type in $R^{3}$ is represented by a closed braid and that such representations are related by some elementary operations called Markov moves. We generalize the notion of a braid to that in 4-dimensional space and establish an analogue of these theorems.
\end{abstract}

\section{INTRODUCTION}

Any link type in $R^{3}$ is represented by a closed braid, and two closed braids represent the same link type if and only if they are related by braid isotopies, stabilizations, and their inverse operations. These facts are well known as Alexander's theorem and Markov's theorem (cf. [B; A; Mo]). The latter is restated as follows: Two braids have ambient isotopic closures in $R^{3}$ if and only if they are related by conjugations, stabilizations, and their inverse operations (Markov moves). These theorems enable braid theory to play an important role in (classical) knot theory (for example, $[J]$ ). We have a natural analogue of them in 4-dimensional space.

There seem to be many generalizations of the notion of classical braids to higher dimensions ([D; G1; G2; BS; MS ], etc.). We use, as a generalization of a classical braid, the notion of a 2-dimensional braid due to O. Viro (cf. [K2]). A similar notion was studied by $L$. Rudolph as a braided surface [R1; R2].

Definition. A 2-dimensional braid (of degree $m$ ) is a compact oriented surface $B$ embedded in bidisk $D_{1}^{2} \times D_{2}^{2}$ such that (1) the restriction of the second factor projection $p_{2}: D_{1}^{2} \times D_{2}^{2} \rightarrow D_{2}^{2}$ to $B$ is an oriented branched covering map of degree $m$ and (2) the boundary of $B$ is a trivial closed braid $X_{m} \times \partial D_{2}^{2} \subset$ $D_{1}^{2} \times \partial D_{2}^{2} \subset \partial\left(D_{1}^{2} \times D_{2}^{2}\right)$, where $X_{m}$ is fixed $m$ points in the interior of $D_{1}^{2}$.

We do not require that the associated branched covering map $B \rightarrow D_{2}^{2}$ is simple, although it is assumed in [K2; R1; R2].

Two 2-dimensional braids are equivalent if they are ambient isotopic by a fiber-preserving isotopy of $D_{1}^{2} \times D_{2}^{2}$, where we regard $D_{1}^{2} \times D_{2}^{2}$ as a $D_{1}^{2}$ bundle over $D_{2}^{2}$, keeping $D_{1}^{2} \times \partial D_{2}^{2}$ fixed. We do not distinguish equivalent 2-dimensional braids. Two 2-dimensional braids are braid isotopic if we can deform one to the other by an isotopy of $D_{1}^{2} \times D_{2}^{2}$ keeping the condition of a 2-dimensional braid and $D_{1}^{2} \times \partial D_{2}^{2}$ fixed.

Let $B$ and $B^{\prime}$ be 2-dimensional braids of the same degree $m$ in $D_{1}^{2} \times D_{2}^{2}$ and in $D_{1}^{2} \times D_{2}^{2^{\prime}}$. Take a boundary connected sum $D_{2}^{2} \natural D_{2}^{2^{\prime}}$ of $D_{2}^{2}$ and $D_{2}^{2^{\prime}}$

Received by the editors May 10, 1993.

1991 Mathematics Subject Classification. Primary 57Q45, 57M25. 
which is also a 2-disk. Then $B \cup B^{\prime}$ in $D_{1}^{2} \times\left(D_{2}^{2} \natural D_{2}^{2^{\prime}}\right)$ forms a 2-dimensional braid. We call it a braid sum of $B$ and $B^{\prime}$ and denote it by $B \cdot B^{\prime}$, which is uniquely determined up to equivalence. The family of equivalence classes of 2-dimensional braids of degree $m$, together with this braid sum operation, is a commutative semi-group. We call it the 2-dimensional braid semi-group of degree $m$.

A 2-dimensional braid $B$ is naturally extended to a closed oriented surface $\widehat{B}$ embedded in $D_{1}^{2} \times S^{2}=D_{1}^{2} \times\left(D_{2}^{2} \cup \overline{D_{2}^{2}}\right)$ with $\widehat{B} \cap D_{1}^{2} \times \overline{D_{2}^{2}}=X_{m} \times \overline{D_{2}^{2}}$. Identifying $D_{1}^{2} \times S^{2}$ with the tubular neighborhood of the standard 2-sphere about the $t$-axis in $R^{4}$, we assume $\widehat{B}$ to be a surface embedded in $R^{4}$ and call it the closure of $B$ (about the $t$-axis) in $R^{4}$ or a closed 2-dimensional braid.

Theorem 1 (Viro [K1]). Any closed oriented surface embedded in $R^{4}$ is ambient isotopic to a closed 2-dimensional braid in $R^{4}$.

In [K1] a closed 2-dimensional braid in $R^{4}$ is treated as a sequence of closed braids in classical dimension, similar ideas to which are found in [G; R1; K3].

Two operations, "conjugations" and "stabilizations", are defined for 2-dimensional braids as a natural analogue of classical ones as follows:

Regard $D_{1}^{2}$ as $I_{1}^{1} \times I_{2}^{1}$ and $X_{m}$ as the set $\left\{z_{1}, \cdots, z_{m}\right\} \subset D_{1}^{2}$ where $I_{i}^{1}$ $(i=1,2)$ is the interval $[-1,1]$ and $z_{i}(i=1, \cdots, m)$ is point $\left(0,1-2^{-i}\right)$.

Let $b$ be a classical braid of degree $m$ in $D_{1}^{2} \times I$ with $\partial b=b \cap D_{1}^{2} \times \partial I=$ $X_{m} \times \partial I$. The product $b \times S^{1}$ is a collection of annuli embedded in $D_{1}^{2} \times I \times S^{1}$. Identify $I \times S^{1}$ with the collar neighborhood $N\left(\partial D_{2}^{2}\right)$ of $\partial D_{2}^{2}$ in $D_{2}^{2}$ so that $\partial D_{2}^{2} \times\{1\}=\partial D_{2}^{2}$ and $D_{1}^{2} \times I \times S^{1}$ with $D_{1}^{2} \times N\left(\partial D_{2}^{2}\right)$.

Up to equivalence, a 2-dimensional braid $B$ is assumed to satisfy that $B \cap$ $D_{1}^{2} \times N\left(\partial D_{2}^{2}\right)$ is the product $X_{m} \times N\left(\partial D_{2}^{2}\right)$. Replace it by $b \times S^{1}$ and we have a 2-dimensional braid. It is called a 2-dimensional braid obtained from $B$ by the conjugation associated with $b$ or the conjugate of $B$ by $b$.

For a nonnegative integer $a$, let $T_{a}$ be a homeomorphism of $D_{1}^{2}=I_{1}^{1} \times I_{2}^{1}$ such that $T_{a}\left(x, y_{i}\right)=\left(x, y_{i+a}\right)$ for each $x \in I_{1}^{1}$ and $y_{i}=1-2^{-i} \in I_{2}^{1}$ $(i=0,1,2, \cdots)$, and let $\widetilde{T_{a}}$ be the homeomorphism of $D_{1}^{2} \times D_{2}^{2}$ which acts on the first factor by $T_{a}$ and on the second factor by the identity. For a 2-dimensional braid $B$ of degree $m, \widetilde{T}_{a}(B)$ satisfies condition (1) of the definition of a 2-dimensional braid and condition (2) replaced $X_{m}$ by $T_{a}\left(X_{m}\right)=$ $\left\{z_{a+1}, \cdots, z_{a+m}\right\}$. Up to equivalence, we may assume that $\widetilde{T}_{a}(B)$ is contained in $I_{1}^{1} \times\left(y_{a}, y_{m+a+1}\right) \times D_{2}^{2} \subset I_{1}^{1} \times I_{2}^{1} \times D_{2}^{2}=D_{1}^{2} \times D_{2}^{2}$. For nonnegative integers $a$ and $b$, let $l_{a}^{b}(B)$ be the union $\left\{z_{1}, \cdots, z_{a}\right\} \times D_{2}^{2} \cup \widetilde{T_{a}}(B) \cup$ $\left\{z_{m+a+1}, \cdots, z_{m+a+b}\right\} \times D_{2}^{2}$, which is a 2-dimensional braid of degree $m+a+b$. $l_{a}^{b}$ is an injection from the 2-dimensional braid semi-group of degree $m$ into that of degree $m+a+b$.

By [K2] the equivalence class of a 2-dimensional braid of degree 2 is determined only by the number of branch points. Let $B^{*}$ be a unique, up to equivalence, 2-dimensional braid of degree 2 with two branch points. It is a unique generator of the 2-dimensional braid semi-group of degree 2. A 2-dimensional braid $B^{\prime}$ of degree $m+1$ is said to be obtained from a 2-dimensional braid $B$ of degree $m$ by a stabilization if $B^{\prime}$ is equivalent to the braid sum $l_{0}^{1}(B) \cdot l_{1}^{0}\left(B^{*}\right)$. 
Theorem 2. Two 2-dimensional braids have ambient isotopic closures in $R^{4}$ if and only if they are related by braid isotopies, conjugations, stabilizations, and their inverse operations.

Remark. A simple 2-dimensional braid is a 2-dimensional braid $B$ whose associated branched covering $B \rightarrow D_{2}^{2}$ is simple. An advanced version of Theorem 1 is found in [K1]: Any closed oriented surface embedded in $R^{4}$ is ambient isotopic to the closure of a simple 2-dimensional braid. Then we have a natural question which is open at present: For two simple 2-dimensional braids with ambient isotopic closures in $R^{4}$, are they related only through simple 2dimensional braids by braid isotopies, conjugations, stabilizations, and their inverse operations?

We work in the piecewise linear category, and surfaces in 4-space are assumed to be embedded properly and locally flatly.

\section{OUTLINE OF THE PROOF}

Our proof follows [B]. Let $\ell$ denote the $t$-axis of $R^{4}$ and $\pi: R^{4} \rightarrow R^{3}$ the projection along $\ell$. We say that an oriented 2-simplex $A$ in $R^{4}$ is in general position with respect to $\ell$ if there is no 3-plane in $R^{4}$ containing both $A$ and $\ell$. Then $\pi(A)$ is an oriented 2-simplex in $R^{3}$ which forms, together with the origin of $R^{3}$, an oriented 3-simplex. Using the orientation of $R^{3}$, we define the sign of $A$ valued in $\{ \pm 1\}$. Theorem 1 is shown as follows: Let $F$ be a closed oriented surface in $R^{4}$ and $K$ a division of $F$, which is a certain kind of tessellation by 2-simplices (not a triangulation but its generalization). We may assume that each 2-simplex of $K$ is in general position with respect to $\ell$. The number of 2 -simplices of $K$ with negative sign is denoted by $h(K)$. If $h(K)=0$, then $F$ is a closed 2-dimensional braid about $\ell$ in $R^{4}$.

Lemma 1 (Sawtooth Lemma). If there is a 2-simplex $A$ of $K$ with negative sign, then there is a sawtooth over $A$ of $F$.

A sawtooth is a family of 3-simplices in $R^{4}$ satisfying a nice condition such that the surgery result of $K$ is a division $K^{\prime}$ of another surface in $R^{4}$ with $h\left(K^{\prime}\right)=h(K)-1$. By this lemma, we can deform $F$ to a closed 2-dimensional braid.

As an analogue of [B], we can define some operations which transform a division of a surface in $R^{4}$ without changing the isotopy type of the surface. A deformation chain is a sequence of divisions of surfaces in $R^{4}$ connected by operations.

Lemma 2. Let $K, K^{\prime}$ be a deformation chain with $h(K)=h\left(K^{\prime}\right) \neq 0$. Then there is a deformation chain $K, K_{1}, \cdots, K_{s}, K^{\prime}$ for some $s \geq 1$ such that $h\left(K_{i}\right)<h(K)$ for $i(i=1, \cdots, s)$.

Lemma 3. Let $K, K^{\prime}, K^{\prime \prime}$ be a deformation chain with $h\left(K^{\prime}\right)>h(K)$ and $h\left(K^{\prime}\right)>h\left(K^{\prime \prime}\right)$. Then there is a deformation chain $K, K_{1}, \cdots, K_{s}, K^{\prime \prime}$ for some $s \geq 1$ such that $h\left(K_{i}\right)<h\left(K^{\prime}\right)$ for $i(i=1, \cdots, s)$.

By the argument in $[\mathrm{B}]$, we have Theorem 2 as a consequence of Lemmas 2 and 3 . The details will appear elsewhere. 


\section{REFERENCES}

[A] J. W. Alexander, A lemma on systems of knotted curves, Proc. Nat. Acad. Sci. U.S.A. 9 (1923), 93-95.

[B] J. Birman, Braids, links and mapping class group, Ann. of Math. Stud., vol. 82, Princeton Univ. Press, Princeton, NJ, 1974.

[BS] E. Brieskorn and K. Saito, Artin Gruppen und Coxeter Gruppen, Invent. Math. 17 (1972), 245-271.

[D] D. Dahm, A generalization of braid theory, Ph.D. thesis, Princeton Univ., Princeton, NJ, 1962.

[G] F. González-Acuña, A characterization of 2-knot groups, preprint.

[G1] D. L. Goldsmith, The theory of motion groups, Michigan Math. J. 28 (1981), 3-17.

[G2] - Motion of links in the 3-sphere, Math. Scand. 50 (1982), 167-205.

[J] V. F. R. Jones, A polynomial invariant for knots via von Neumann algebras, Bull. Amer. Math. Soc. (N.S.) 12 (1985), 103-111.

[K1] S. Kamada, A characterization of groups of closed orientable surfaces in 4-space, Topology 33 (1944), 113-122.

[K2] Surfaces in $R^{4}$ of braid index three are ribbon, J. Knot Theory Ramifications 1 (1992), 137-160.

[K3] _ 2-dimensional braids and chart descriptions, Topics in Knot Theory, Proceedings of the NATO ASI on Topics in Knot Theory, Turkey, 1992 (M. E. Bozhüyük, ed.), pp. 277-287.

[MS] Yu. I. Manin and V. V. Schechtman, Arrangements of hyperplanes, higher braid groups and higher Bruhat orders, Adv. Stud. Pure Math., vol. 17, Academic Press, Boston, MA, 1986, pp. 289-308.

[Mo] H. R. Morton, Threading knot diagrams, Math. Proc. Cambridge Philos. Soc. 99 (1986), 247-260.

[R1] L. Rudolph, Braided surfaces and Seifert ribbons for closed braids, Comment. Math. Helv. 58 (1983), 1-37.

[R2] _ Special positions for surfaces bounded by closed braids, Rev. Mat. Iberoamericana 1 (1985), 93-133.

Department of Mathematics, Osaka City University, Sumiyoshi, Osaka 558, Japan

E-mail address: g54042@jpnkudpc.bitnet 